\title{
-NOTES-
}

\section{THE OSCILLATIONS OF A VISCOUS LIQUID DROP*}

\author{
BY W. H. REID (Brown University)
}

1. Introduction. In his discussion of the effect of viscosity on the small oscillations of a liquid globe, Lamb [1, pp. 639-641] observed that the results obtained in the limiting case of 'small viscosity' are independent of the nature of the forces which produce the tendency to the spherical form. When these forces are due to self-gravitation, the problem has been solved completely by Lamb [2] and Chandrasekhar [3] for arbitrary values of the viscosity, and the latter author has given detailed numerical results for the aperiodic modes of decay for $l=1,2,3$ and 4 , where $l$ is the order of the spherical harmonic deformation considered.

The question then arises whether or not these more general results for arbitrary values of the viscosity are also independent of the nature of the forces which produce the tendency to the spherical form. While no attempt will be made to answer this question in complete generality, it will be shown that when these forces are due to surface tension the results obtained are identical with those obtained by Lamb and Chandrasekhar for a self-gravitating globe.

2. Statement of the problem. We consider a liquid drop which, in the undisturbed state, will be spherical under the action of surface tension forces. If the external pressure is taken to be zero, then the internal pressure will have the constant value

$$
p=2 T_{1} / R,
$$

where $T_{1}$ is the surface tension per unit length and $R$ is the radius of the sphere. To study the oscillations of this configuration we consider a deformation of the liquid surface of the form

$$
r=R\left[1+\epsilon_{l}^{m}(\theta, \varphi)\right]
$$

where $Y_{l}^{m}(\theta, \varphi)=P_{l}^{m}(\theta) e^{-i m \varphi}$ is a surface harmonic of the first kind and $\epsilon \ll 1$. In the absence of viscosity, assuming a time dependence $\epsilon=\epsilon_{0} e^{i \sigma t}$, the frequencies of oscillation are $[1$, p. 475$]$

$$
\sigma_{l ; 0}^{2}=l(l-1)(l+2) \frac{T_{1}}{\rho R^{3}},
$$

and are the same for all values of $m$.

When viscosity is included, the departures from the equilibrium state are then governed by the linearized equations of motion

$$
\frac{\partial \mathbf{u}}{\partial t}=-\operatorname{grad} \frac{\delta p}{\rho}-\nu \operatorname{curl}^{2} \mathbf{u} \text { and } \operatorname{div} \mathbf{u}=0
$$

*Received January 29, 1959. This work was sponsored by the Office of Naval Research under Contract Nonr 562(07). 
On taking the divergence of this equation we have $\nabla^{2}(\delta p / \rho)=0$; accordingly, the first order change in the pressure in the sphere is

$$
\delta p / \rho=\epsilon P_{0} x^{l} Y_{l}^{m},
$$

where $x=r / R$ and $P_{0}$ is a constant of integration that must be determined later from the boundary conditions. Equation (4) must now be solved for $\mathbf{u}$ as an inhomogeneous equation with $\delta p / \rho$ given by Eq. (5). In treating the viscous case, it is convenient to take the time dependence in the form $\epsilon=\epsilon_{0} e^{-\sigma t}$ so that the real part of $\sigma$ will be always positive.

Since $\operatorname{grad}(\delta p / \rho)$ is a poloidal divergence-free vector, it can be represented in terms of a single defining scalar function $\Pi(x)$; in this representation its $r$-component is given by

$$
\left(\operatorname{grad} \frac{\delta p}{\rho}\right)_{r}=\epsilon_{0} \sigma^{2} R \frac{\Pi(x)}{x^{2}} Y_{l}^{m} e^{-\sigma t},
$$

where

$$
\Pi(x)=\Pi_{0} x^{l+1} \quad \text { and } \quad \Pi_{0}=\left(l / \sigma^{2} R^{2}\right) P_{0} .
$$

Since $\operatorname{grad}(\delta p / \rho)$ is a purely poloidal vector, it follows that $\mathbf{u}$ must also be purely poloidal and, accordingly, it too can be represented in terms of a single defining scalar function $U(x)$; its $r$-component can then be written in the form

$$
u_{r}=\epsilon_{0} \sigma R \frac{U(x)}{x^{2}} Y_{l}^{m} e^{-\sigma t} .
$$

When defined in this manner, both $\Pi(x)$ and $U(x)$ are dimensionless. The dynamical equation for $U(x)$ which then follows from Eq. (4) is

$$
\left[\frac{d^{2}}{d x^{2}}-\frac{l(l+1)}{x^{2}}+q^{2}\right] U(x)=q^{2} \Pi(x),
$$

where

$$
q^{2}=\sigma R^{2} / \nu
$$

3. Boundary conditions. At the deformed surface of the drop the radial component of the velocity must be equal to the velocity of the surface itself and this requirement leads to the kinematical boundary condition

$$
U=-1 \text { at } x=1 .
$$

The other boundary conditions are obtained by considering the behavior of the viscous stresses at the surface. Thus, the requirement that the tangential stresses vanish at the surface leads to the single boundary condition

$$
\left[\frac{d^{2}}{d x^{2}}-\frac{2}{x} \frac{d}{d x}+\frac{l(l+1)}{x^{2}}\right] U=0 \quad \text { at } \quad x=1 .
$$

These two boundary conditions are clearly independent of the nature of the forces which tend to maintain the spherical form, but the third boundary condition, which 
follows from considering the normal component of the viscous stress, is not. This stress component is given by

$$
-p_{r r}=p+\delta p-2 \mu \frac{\partial u_{r}}{\partial r}
$$

and the condition to be imposed at the surface is

$$
-p_{r r}=T_{1}\left(\frac{1}{R_{1}}+\frac{1}{R_{2}}\right),
$$

where $R_{1}$ and $R_{2}$ are the principal radii of curvature, reckoned positive when directed inwards. For a surface deformed in the manner (2), we have [1, p. 475]

$$
\frac{1}{R_{1}}+\frac{1}{R_{2}}=\frac{1}{R}\left[2+(l-1)(l+2) \epsilon Y_{l}^{m}\right] .
$$

The third boundary condition can therefore be written in the form

$$
(l-1)(l+2) \frac{T_{1}}{\rho R}=P_{0} x^{l}-2 \nu \sigma \frac{d}{d x}\left(\frac{U}{x^{2}}\right) \text { at } x=1 .
$$

The solution of Eq. (9) subject to the boundary conditions (11), (12) and (16) then leads to a characteristic equation for $\sigma$ in terms of the physical parameters of the problem.

4. Solution and results. The derivation of the characteristic equation for $\sigma$ is not difficult but can be entirely avoided in the following manner. Observe that the dependence of the boundary condition (16) on $T_{1}$ is more apparent than real; for, if we let

$$
\alpha^{2}=\frac{\sigma_{l ; 0} R^{2}}{\nu} \text { so that } \frac{\sigma_{l ; \nu}}{\sigma_{l ; 0}}=\frac{q^{2}}{\alpha^{2}},
$$

then this third boundary condition can be written in the form

$$
\alpha^{4}=q^{2}\left\{q^{2} \Pi_{0}-2 l\left[U^{\prime}(1)-2 U(1)\right]\right\}
$$

and this relation no longer contains any physical parameters of the problem. It is, in fact, identical with the corresponding boundary condition for a self-gravitating sphere; the solution, in terms of $\alpha$ and $g$, must therefore be identical with the one given by Lamb and Chandrasekhar:

$$
\frac{\alpha^{4}}{q^{4}}+1=\frac{2(l-1)}{q^{2}}\left[l+(l+1) \frac{q-2 l Q_{l+1 / 2}(q)}{q-2 Q_{l+1 / 2}(q)}\right],
$$

where

$$
Q_{l+1 / 2}(q)=J_{l+3 / 2}(q) / J_{l+1 / 2}(q)
$$

and the $J$ 's are spherical Bessel functions. For $q \rightarrow \infty$, the limiting case of 'small viscosity', we recover Lamb's result

$$
\sigma_{l ; \nu}=(l-1)(2 l+1) \nu / R^{2} \pm i \sigma_{l ; 0} .
$$

Thus, the physical forces which maintain the spherical form enter only in the definition of the inviscid modes $\sigma_{l ; 0}$ and these, as we have seen, can be completely eliminated by a suitable choice of dimensionless parameters.

The characteristic equation (19) admits solutions of two types. For values of $\alpha^{2}$ 
which exceed a certain critical value, damped oscillations will occur, but for values of $\alpha^{2}$ less than this critical value, two aperiodic modes of decay appear. The solution of Eq. (19) at this critical point for the principal mode $l=2$ is [3]

$$
\sigma_{2 ; 0} R^{2} / \nu=3.69 \text { and } \sigma_{2 ; \nu} / \sigma_{2 ; 0}=0.968 \text {. }
$$

For a drop of water surrounded by air $\left(T_{1}=74\right.$ dynes $\left./ \mathrm{cm}\right)$ this gives a radius $R=0.23$ $\mathrm{mm}$. Drops larger than this critical radius will therefore tend to oscillate while smaller drops will be aperiodically damped.

\title{
REFERENCES
}

1. H. Lamb, Hydrodynamics, 6th ed., Cambridge University Press, 1932

2. H. Lamb, On the oscillations of a viscous spheroid, Proc. London Math. Soc. (1), 13, 51-66 (1881)

3. S. Chandrasekhar, The oscillations of a viscous liquid globe, Proc. London Math. Soc. (3), 9, 141-149 (1959)

\section{ON THE DIFFRACTION OF AN ARBITRARY PULSE BY A WEDGE OR A CONE*}

\author{
BY LU TING (Polytechnic Institute of Brooklyn)
}

\begin{abstract}
By virtue of Green's Theorem, it is shown that for the diffraction of an arbitrary two-dimensional incident pulse by a wedge of angle $\mu$, the ratio of the resultant velocity potential to the corresponding value of the incident pulse at the corner of the wedge at any instant is equal to $2 \pi /(2 \pi-\mu)$; and that for the diffraction of a threedimensional pulse by a cone of solid angle $\omega$, the ratio at the vertex of the cone is equal to $4 \pi /(4 \pi-\omega)$.
\end{abstract}

Two-dimensional space. The statement concerning diffraction of a pulse by a wedge is evidently true in the special case of an incident plane Heaviside pulse which was solved by Keller and Blank [1]. It therefore also follows for all incident pulses which are superpositions of plane Heaviside pulses, or limits of such superpositions. Since this includes all incident pulses it yields the preceding statement. However, these considerations depend upon knowing the exact solution in a special case which the following proof does not require.**

Let $t=0$ be the instant at which the incident pulse $\varphi^{(i)}$ hits the corner of the wedge, which is located at the origin $\left(x_{1}=0, x_{2}=0\right)$. Let $h\left(x_{1}, x_{2}\right)$ and $k\left(x_{1}, x_{2}\right)$ denote, respectively, $\varphi^{(i)}$ and $\varphi_{t}^{(i)}$ at an instant $t=-t_{0}<0$ if the corner is absent. If $G$ represents the domain in the $x_{1}-x_{2}$ plane outside which both $h$ and $k$ vanish, then the origin must lie outside $G$. When the wedge is present, the region $G$ lies outside the wedge if the incident disturbance $\varphi^{(i)}$ has not hit either side of the wedge at $t=-t_{0}<0$. Then the resultant disturbance $\varphi$ at any instant $t_{1}>-t_{0}$ fulfills the wave equation and the same initial conditions as that of $\varphi^{(i)}$, i.e., in the region exterior to the wedge

$$
\varphi\left(-t_{0}, x_{1}, x_{2}\right)=h\left(x_{1}, x_{2}\right) \text { and } \frac{\partial \varphi}{\partial t}\left(-t_{0}, x_{1}, x_{2}\right)=k\left(x_{1}, x_{2}\right) .
$$

*Received Feb. 10, 1959; revised manuscript received May 27, 1959.

**This paragraph is based on a private communication from Prof. J. B. Keller, Institute of Mathematical Sciences, New York University. 